\title{
Substation protection method for missing measurement information of transformer and bus connecting line
}

\author{
Liu Wei ${ }^{1}$, Wang Yuxue ${ }^{1}$, Wang Zengchao ${ }^{1}$, Xu Zhile $^{2}$, Li Yiquan ${ }^{1}$, Deng Xuyang ${ }^{1}$, Liu Shidan ${ }^{1}$, Liu Kun ${ }^{1}$, Wen Minghao ${ }^{2}$ \\ ${ }^{1}$ Power Dispatching Control Center of Guangdong Power Grid, Guangzhou 510600, China \\ ${ }^{2}$ Huazhong University of Science and Technology, Wuhan 430074, China
}

\begin{abstract}
In order to solve the problems of protection misoperation and refusing to operate caused by the lack of measurement information in smart substation, a substation protection method which can effectively eliminate the influence of transformer inrush current and bus CT saturation when the measurement information of substation and bus connecting line is missing is proposed. Through the theoretical analysis of current synthesis method based on Kirchhoff current law, it is proved that transformer inrush current and bus CT saturation will lead to the misjudgment of differential protection. Accordingly a new method of station protection is proposed to deal with the lack of measurement information. In order to verify the correctness of the method, taking a typical $220 \mathrm{kV}$ substation model as an example, PSCAD / EMTDC electromagnetic transient simulation software is used to simulate and verify different conditions and different types of faults. The results show that the proposed protection method can accurately eliminate the interference of transformer inrush current and bus CT saturation, ensure the correct action of differential protection, and improve the stability of substation when the measurement information is missing.
\end{abstract}

\section{Introduction}

Substation is one of the basic links of power grid, and it is the source of basic operation data collection and command execution unit of power grid. Based on the architecture of digital substation, intelligent substation realizes the unified information collection, transmission, analysis and processing platform of the whole station, which enables the substation to have advanced functions such as automatic operation control, equipment condition maintenance, operation state adaptation, intelligent analysis and decision-making, etc. Once the measurement information is missing, it will cause serious interference to the stability of the power system. The lack of measurement information mainly refers to the situation that the measuring element can not collect the system voltage and current due to fault. If the measurement information is missing, the longitudinal protection and other main protections will not act correctly due to the lack of information, thus increasing the risk and hidden danger of system failure. And for the lack of measurement information, we can only rely on the power dispatching center to check the components that may fail one by one, which will lead to a large area of power system blackout and unable to restore power supply in time, causing a lot of inconvenience to production and life. The importance of complete and accurate substation measurement information is self-evident, but in the practical situation, there are often many unpredictable situations. Therefore, it is very important and urgent to study a relay protection to deal with the lack of substation measurement information.

In the first line of defense of power system, the performance of relay protection is of great significance to the safe and stable operation of the whole power grid. However, in recent years, with the expansion of the scale of power system and the complexity of operation mode, relay protection relying on local information is becoming more and more inadequate. For example, in the case of lack of measurement information, relay protection is difficult to accurately identify the fault; the coordination between different relay protection settings is difficult; in the case of power flow transfer, the protection may trip, leading to a large area outage Electrical accidents, etc. In order to solve these problems, based on the development of optical fiber communication technology and information sharing technology in intelligent substation, relevant experts put forward the concept of substation protection. Because the substation protection can obtain the multi-source information in substation in real time, it can fundamentally improve the performance of backup protection, and formulate the appropriate tripping strategy, which can quickly and selectively remove the faults that are difficult to distinguish.

In order to solve the problem that traditional relay protection is difficult to accurately identify the fault when the measurement information is missing, scholars at home and abroad put forward a series of methods and strategies to improve the protection. In reference [1], a regional protection scheme based on logic information is proposed. According to the configuration of circuit breaker, the 
regional protection range is divided, and combined with the action information of III section directional current protection, the operation criterion of regional protection is formed, so that the fault location can be determined in the case of missing information. Reference [2-4] proposes a series of protection schemes based on station information, which can deal with the situation of lack of substation information. However, the above scheme only uses the information of substation area, and it is difficult to deal with all the information missing faults. Reference [5-10] proposes a series of wide area protection schemes based on multi-source information. Although it can effectively solve the problem of missing information in substation measurement points, the principle of the protection scheme proposed in reference [5-9] is more complex, which is difficult to realize in practical engineering application. The method proposed in reference [10] does not consider the situation of continuous missing information in multiple measurement points. To sum up, although some progress has been made in recent years, some special conditions have not been considered, such as ignoring the impact of transformer inrush current and bus CT saturation, which will lead to some failure situations that can not be dealt with, so further in-depth research is needed.

Based on the centralized substation protection system, this paper analyzes the influence of transformer inrush current and bus CT saturation on relay protection in detail, and proposes a substation protection method to deal with the lack of measurement information. This method can eliminate the influence of inrush current and CT saturation to achieve the backup protection function. The principle is simple, and it can adapt to various power grid structures, and is easy to implement in engineering. Then, a typical $220 \mathrm{kV}$ substation model is used for simulation verification, which shows that the proposed new substation protection method can effectively deal with inrush current and bus CT saturation under the condition of missing measurement information, and can improve the stability of power system.

\section{Theoretical analysis}

\section{1 the synthetic current to deal with the lack of measurement information}

Because the failure of transformer and bus in the same substation is a small probability event, the simultaneous failure of transformer and bus is not considered. For the missing measurement information of transformer and bus connecting line, in general, the current information of missing measuring point is replaced by the current information of its adjacent measuring point by Kirchhoff current law. As shown in Figure 1, if the measurement information on the connection line of three winding transformer and bus is missing, the current information of all adjacent measuring points of the missing measurement point is obtained, assume that the measurement information of point $l$ of transformer 1 is missing, $\overrightarrow{I_{T 1}^{\prime}}$ and $\overrightarrow{I_{T 2}^{\prime}}$ are the current of the two branches $J$ and $K$ on transformer 1 and are converted to the high voltage side, $\vec{I}_{B i}$ is the current information of the branch other than the branch where the measurement information is missing from $220 \mathrm{kV}$ bus, among which $1 \leq i \leq N-1, N$ is the number of branch lines on the bus.

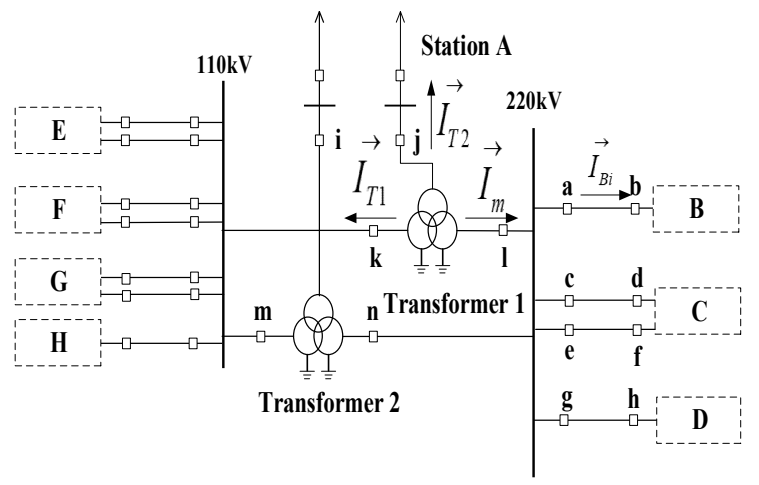

Fig. 1. Substation diagram

According to the current information of the measuring points on the other branches of the transformer except the branch with missing measuring information, the current information $I_{m 1}$ of the measuring points with missing information is synthesized:

$$
\overrightarrow{I_{m 1}}=-\left(\overrightarrow{I_{T 1}^{\prime}}+\overrightarrow{I_{T 2}^{\prime}}\right)
$$

$\overrightarrow{I_{m 1}}$ can be used to replace the current information of the missing measuring point, and then judge the bus differential protection. If the criterion is met, it means that the transformer or bus is in fault.

In the same way, according to the current information of the measuring points on the bus except for the branch with missing measuring information, the current information $\overrightarrow{I_{m 2}}$ of the measuring points with missing information is synthesized

$$
\overrightarrow{I_{m 2}}=\sum_{i=1}^{N-1} \overrightarrow{I_{B i}}
$$

$\overrightarrow{I_{m 2}}$ can be used to replace the current information of the missing measuring points, and then judge the transformer differential protection. If the criterion is met, it means that the transformer or bus is in fault.

The above method of synthesizing current essentially combines the current information of all normal measuring points adjacent to the missing measuring point to form a differential protection.

$$
\left|\overrightarrow{I_{T 1}^{\prime}}+\overrightarrow{I_{T 2}^{\prime}}+\sum_{i=1}^{N-1} \overrightarrow{I_{B i}}\right|>I_{\text {set }}
$$

$I_{\text {set }}$ is the setting value of differential protection. If the differential protection meets the criterion, it indicates that the fault is located in the fault area. Then, disconnect 
the circuit breaker of the connecting line, and use the current information of the branches on both sides to judge the transformer and bus differential protection, so as to determine whether the fault is on the transformer or bus, so as to effectively remove the fault.

\subsection{Influence of transformer inrush current on synthetic current}

According to the analysis of the mechanism and characteristics of inrush current, it can be seen that in the practical operation, because the transformer is a non-linear load, a large inrush current will be generated when the transformer is put into the power grid after no-load closing or when the external fault of the transformer is cut off and the normal operation is restored, which will cause the iron core to be over saturated. This inrush current will make $\overrightarrow{I_{m 1}}$ in the above method formula (1) can not replace the current at the missing measurement information correctly, and the unbalanced current increases, which leads to the misjudgment of large differential protection. At the same time, the inrush current is full of high harmonic and DC components, which will cause the power quality to decline, and may cause resonance overvoltage in serious cases.

\subsection{Influence of bus CT saturation on synthetic current}

Current transformer is the measurement equipment of power system, which can convert the large current of primary system into small current of secondary system in proportion. Because the core magnetic saturation is the essential cause of current transformer saturation, the causes of current transformer saturation can be divided into two categories: one is the steady-state saturation caused by large amplitude steady-state symmetrical current, the other is the transient saturation caused by aperiodic component and core remanence. There are many factors that affect the saturation of the current transformer, and the accuracy of the measurement of the bus current transformer directly determines whether the relay protection device can operate correctly. Under normal conditions, the measurement error of current transformer is very small, and it can be approximately considered that the primary and secondary side current is transferred linearly, which can ensure the correct action of the protection device; under fault conditions, the measurement error of current transformer may be large, and the output secondary current is far away from the true value, which may lead to protection misoperation, delay action or refusal action.

When a short circuit occurs in the line outside the bus and close to the bus, the current of all the non fault active lines connected to the bus will flow to the fault line. The current transformer installed in the fault line may be saturated under the action of large short-circuit current, resulting in the distortion of the secondary current waveform and the large unbalanced current in the differential circuit. If the current transformer on one side of an outgoing line on the bus is seriously saturated or the saturation degree on both sides is inconsistent, the current in the missing measurement information cannot be replaced correctly by $\overrightarrow{I_{m 2}}$ in the formula (2) of the above method, resulting in misjudgment of differential protection.

In view of the shortcomings of the above methods, this paper proposes a new station protection method which can eliminate the influence of transformer inrush current and bus CT saturation, and deal with the lack of measurement information of transformer and bus connecting line.

\section{A new method of substation protection to deal with the lack of measurement information}

Based on the above analysis, this paper proposes a backup protection scheme for substation with missing measurement information, which can eliminate the influence of transformer inrush current and bus CT saturation. After the fault occurs, the substation area protection device can obtain the measurement information of each measurement point according to the topology structure of the substation.

1) Detect whether the measurement information of transformer and bus connecting line is missing in the substation.

2) In order to judge whether there is a fault in the substation area, the current information of transformer and bus except the connecting line is combined to form a differential protection:

$$
\left|\sum_{j=1}^{m} I_{j}\right|>I_{c d z d}
$$

$I_{c d z d}$ is the starting setting value of differential current, the calculation of $I_{j}$ includes all branch currents of transformer and bus except the connecting line (convert to the same side). If the differential current meets the criterion, the influence of inrush current and saturation should be further eliminated.

3) The second harmonic braking principle is used to identify inrush current:

$$
I_{2 n d}>K_{2 x b} \times I_{1 s t}
$$

$I_{2 n d}$ is the effective value of the second harmonic in the current, $I_{1 s t}$ is the effective value of the fundamental wave in the current, $K_{2 x b}$ is the setting value of the second harmonic braking coefficient, and its value is generally $0.15 \sim 0.2$. If the criterion is not satisfied, the time difference method is used to detect whether the bus $\mathrm{CT}$ is saturated.

4) Time difference method is used to identify whether the bus CT is saturated or not, and the time difference between fault occurrence time and differential current occurrence time is calculated:

$$
t_{b l c d}-t_{d i}>\Delta t
$$

$t_{d i}$ is the operation time of power frequency variation 
current element, $t_{b l c d}$ is the operation time of power frequency variation differential element, $\Delta t$ is the fixed time, and the selection of its value determines the CT saturation depth, generally taking $2 \sim 3 \mathrm{~ms}$, which can meet the requirements of rapid action of fault in the area and reliable identification of CT saturation of fault outside the area. If the criterion is not met, the tripping command is sent to the circuit breaker of the connecting line between the transformer and the bus.

5) When the connecting lines of transformer and bus are disconnected, the current information of other branches of bus except the connecting lines is used to judge the bus differential protection:

$$
\left\{\begin{array}{l}
|| \sum_{j=1}^{m} I_{j} \mid>I_{c d z d} \\
|| \sum_{j=1}^{m} I_{j}\left|>K \sum_{j=1}^{m}\right| I_{j} \mid
\end{array}\right.
$$

$K$ is the ratio braking coefficient, $I_{c d z d}$ is the starting fixed value of the differential current. The calculation of $I_{j}$ includes all branch currents except the connecting line. If the differential current meets the criterion, the bus fails, and the protection sends a trip command to the other branches of the bus except the connecting line, and the protection acts to trip the circuit breakers on the other branches of the bus except the connecting line.

6) Based on the current information of other branches except the connecting line, the differential protection of transformer is judged:

$$
\left|\sum_{k=1}^{n} I_{k}\right|>I_{s e t}
$$

$I_{\text {set }}$ is the setting value of the differential current, and the calculation of $I_{k}$ includes all the branch currents of the transformer except the connecting line (convert to the same side). If the differential current meets the criterion, the transformer fails, and the protection will send a trip command to the other branches except the connecting line of the transformer, and the protection will trip the circuit breakers on the other branches except the connecting line of the transformer.

To sum up, the flow chart of the new substation protection method with missing measurement information is shown in Figure 2.

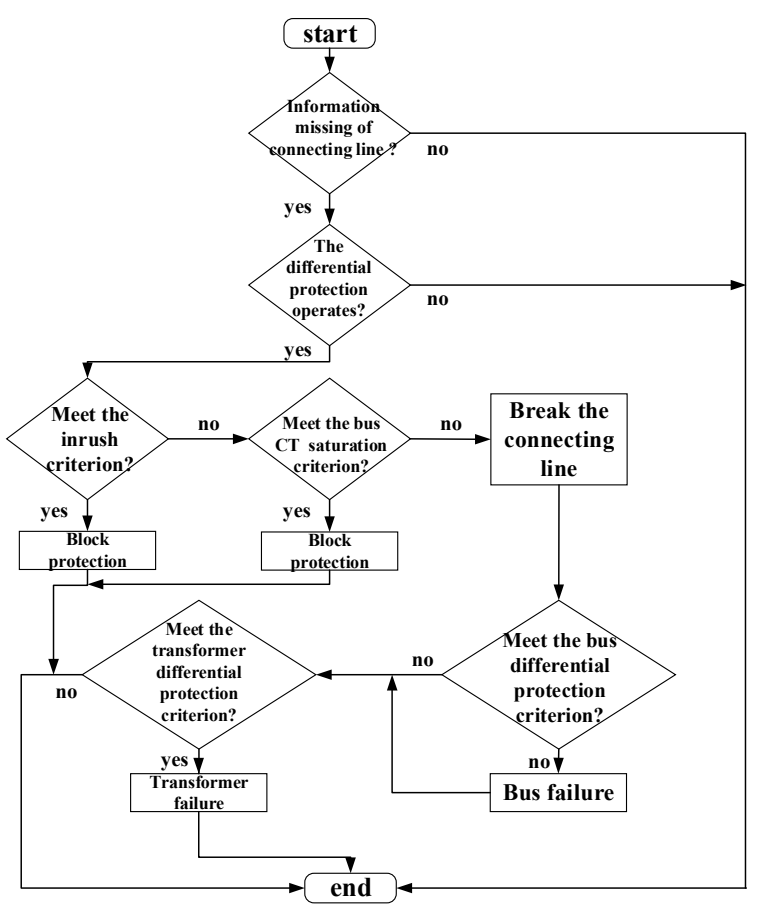

Fig. 2. Backup protection process of substation with missing information

\section{Simulation verification}

In order to verify the performance of the above substation protection method, this paper takes a $220 \mathrm{kV}$ substation as an example for analysis, and its topology is shown in Figure 3. In the electromagnetic transient simulation software PSCAD / EMTDC, the system model is built, and the simulation verification is carried out in different fault locations and different working conditions.

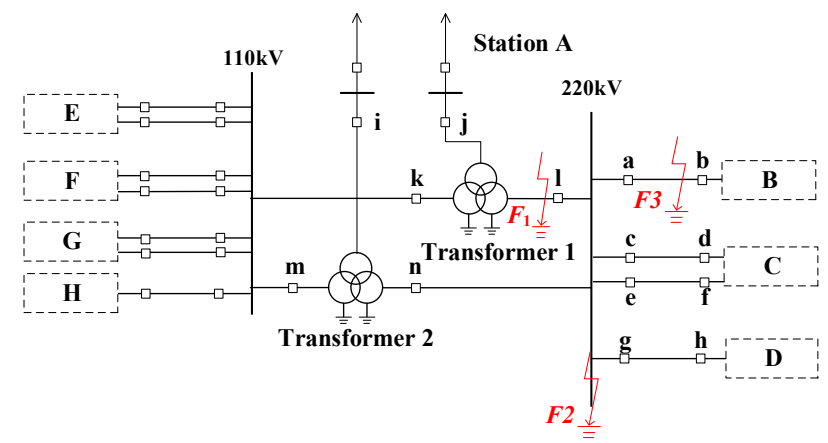

Fig. 3. Substation topology

\subsection{Simulation analysis without transformer inrush current and bus CT saturation when information is missing}

When $\mathrm{t}=0.3 \mathrm{~s}$, A-phase grounding fault $F_{l}$ occurs at $l$ outlet of transformer1's high-voltage side, and there is no transformer inrush current and bus CT saturation, it can be seen from Figure 4 that the differential current of the differential protection formd by the current information of transformer and bus except the connecting line increases rapidly, and exceeds the starting setting value (the starting setting value of the model is set to $10 \mathrm{~A}$ ), the differential 
protection acts, and the fault in the area can be judged, and then the connecting line $l$ between the transformer and the bus is disconnected (i.e. the branch with missing measurement information).

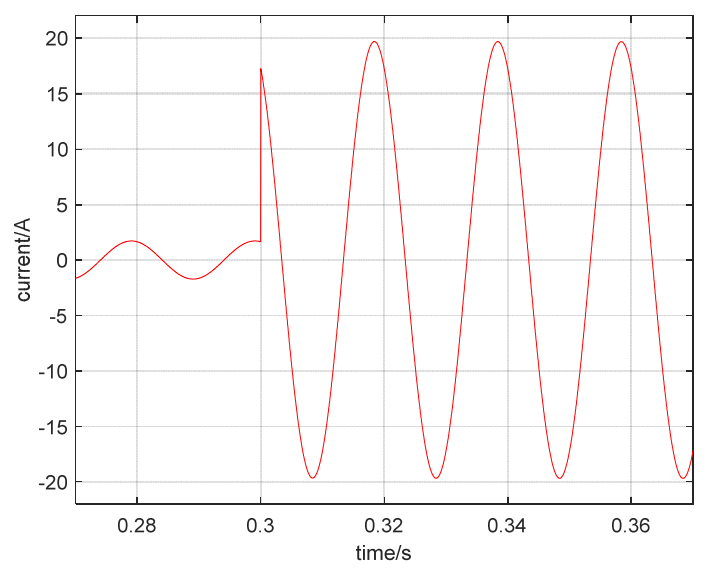

Fig. 4. Differential current of the differential protection

After the judgment of bus differential protection, we can see that the differential current of bus differential protection fluctuates slightly due to the influence of unbalanced current, but the current is very weak, which will not cause the misoperation of bus differential protection.

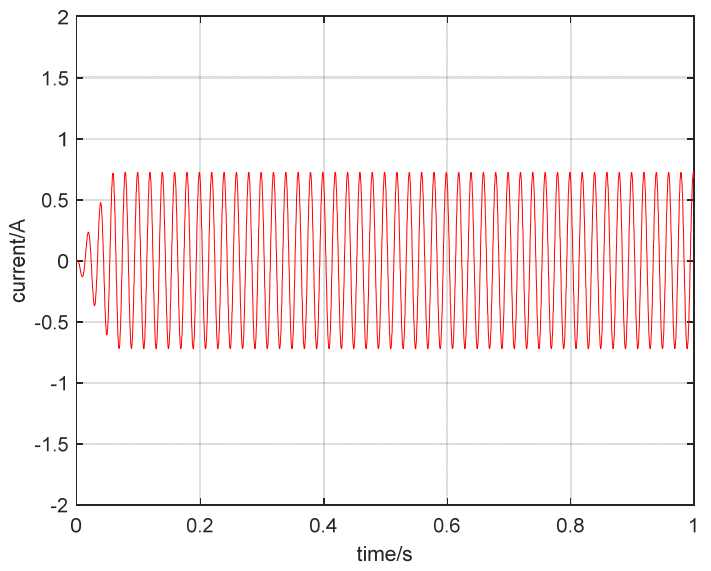

Fig. 5. Differential current of bus differential protection

After judging the transformer differential protection, we can see that after $\mathrm{t}=0.3 \mathrm{~s}$, the differential current increases rapidly and exceeds the threshold value (for the model, the starting setting value is set to $3 \mathrm{~A}$ ), the transformer differential protection acts, the $k$ and $j$ branches are disconnected, and the fault is removed reliably.

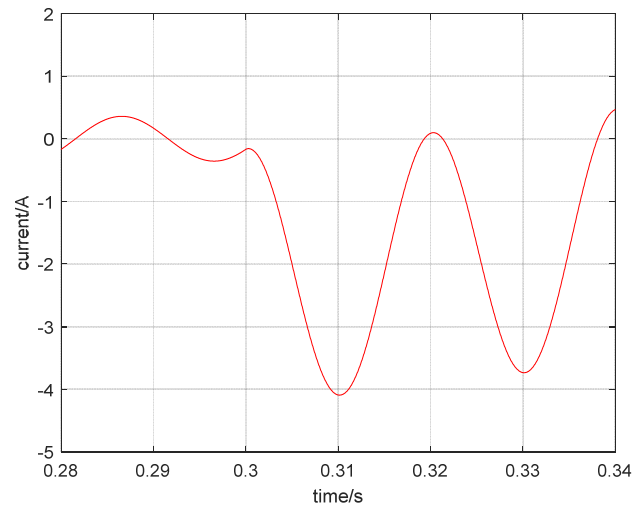

Fig. 6. Differential current of transformer differential protection

\subsection{Simulation analysis of transformer inrush current when information is missing}

When the transformer no-load switch-on or external fault clearing voltage recovery, the transformer excitation branch will produce a large transient excitation current, which can reach $6 \sim 8$ times of the rated current. In this paper, through the simulation of transformer no-load switch-on at $\mathrm{t}=0.1 \mathrm{~s}$, the transformer inrush current waveform is obtained as shown in Figure 7.

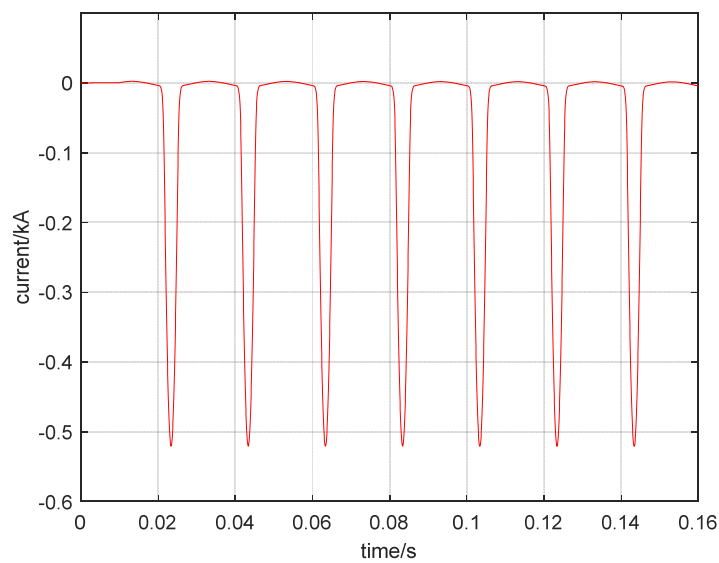

Fig. 7. Inrush current waveform of transformer

It can be seen that because the waveform is only biased to one side of the time axis, it belongs to unidirectional inrush current and lasts for a long time, it will form a large unbalanced current in the differential circuit, which will seriously affect the normal operation of the differential protection.

The FFT module in PSCAD is used for harmonic analysis of inrush current. It can be seen that the harmonic content of each order is generally large, and the harmonic content gradually decreases with the increase of harmonic number. The specific content of the first seven harmonics is shown in Figure 8. 


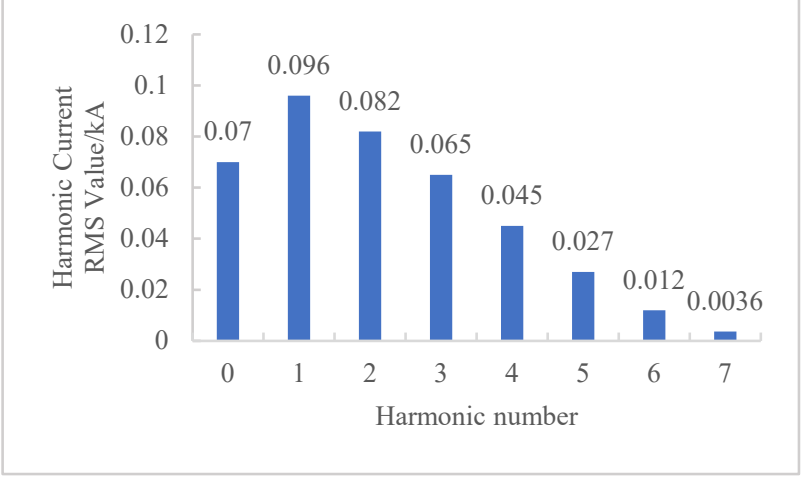

Fig. 8. Harmonic content of inrush current

In practical engineering, $K_{2 x b}$ is usually set to 0.2 :

$$
0.082>0.2 \times 0.096
$$

At this time, the second harmonic content meets the blocking condition, and the differential protection should be blocked.

Therefore, in order to make the differential protection have certain reliability and selectivity, it is necessary to avoid the influence of inrush current on transformer differential protection. The usual way is to rely on various discriminant conditions to determine inrush current and reliably block the differential protection, such as using secondary harmonic restraint, the principle of waveform symmetry, using differential relay with fast saturation core, etc.

\subsection{Simulation analysis of bus CT saturation when information is missing}

During external faults of the bus, the current of all the nonfault active lines connected to the bus will flow to the fault line, which will produce a great fault current, and the fault current may contain a large non periodic component. Due to the nonlinearity of the current transformer core, the current transformer may quickly enter a serious saturation state after a period of time, at this time, the CT will be out of service If the primary current can not be transformed, the waveform of the secondary current will be distorted. the current transformer based on JA model in PSCAD is used. When $\mathrm{t}=0.2 \mathrm{~s}$, the single-phase ground fault outside the bus area is set. The CT saturation waveform on the bus fault branch obtained by simulation is shown in Figure 9.

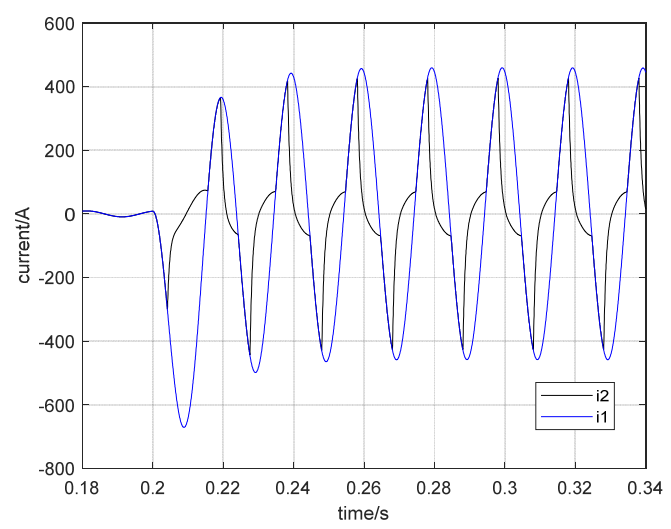

Fig. 9. Bus CT saturation waveform
In Figure 9, the blue line $i_{1}$ and the black line $i_{2}$ are the primary and secondary currents respectively. It can be seen that when the core reaches the saturation state, there is a large error between the primary and secondary currents, resulting in that the primary current can no longer be linearly transferred by CT, which will cause the protection to fail to work normally to a great extent.

It can be seen from the above formula (6) that the effective anti-saturation criterion can be obtained by using the time difference method. In practical engineering, considering the sensitivity and certain margin of the criterion, the fixed time $\Delta t$ is generally set to $2 \mathrm{~ms}$. It can be seen from Figure 10 that when $\mathrm{t}=0.2 \mathrm{~s}$, the singlephase grounding short circuit fault occurs outside the bus area, which is different from the fault in the area that the power frequency variation differential element and the power frequency variation current element basically act at the same time. Because the fault starting CT has not entered saturation, the two actions are not synchronous. The action of the differential element will lag behind the current element by about $4.2 \mathrm{~ms}$. The time difference meets the criterion, and the differential protection should be blocked. This feature can effectively eliminate the influence of bus CT saturation.

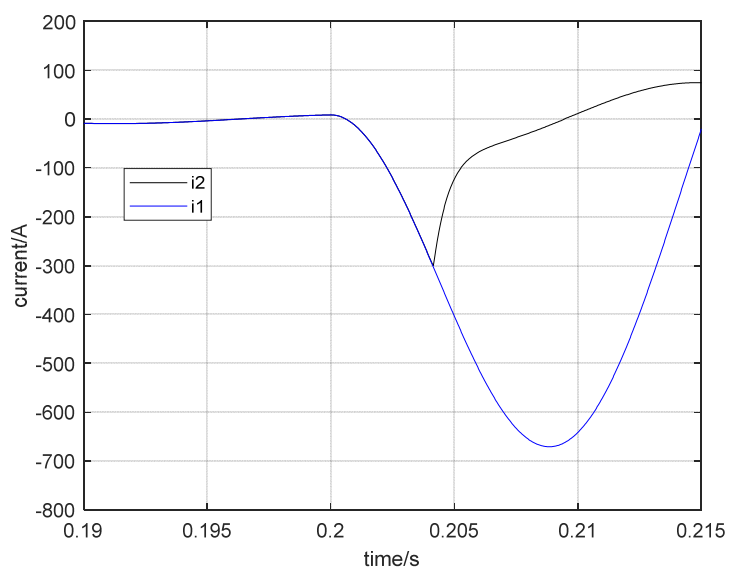

Fig. 10. Status of bus CT before and after fault

It can be seen that the differential protection with ratio braking characteristic can effectively avoid the steadystate error caused by CT, but the transient error caused by CT is much larger than the steady-state error in the transient process of system fault. Therefore, the bus current transformer must consider the proprietary anti CT saturation measures to slow down or avoid the disadvantages caused by CT transient saturation influence.

\subsection{Comprehensive simulation analysis}

When the measurement information of transformer and bus connecting line is missing, considering different working conditions and different types of faults, the action results of substation protection are shown in Table 1. 
Table 1. Results of substation protection action when different types of faults occur

\begin{tabular}{ccccccc}
$\begin{array}{c}\text { Fault } \\
\text { location }\end{array}$ & $\begin{array}{c}\text { Inrush } \\
\text { current }\end{array}$ & $\begin{array}{c}\text { CT } \\
\text { saturation }\end{array}$ & $\begin{array}{c}\text { Whether the } \\
\text { differential } \\
\text { protection } \\
\text { operates or } \\
\text { not }\end{array}$ & $\begin{array}{c}\text { Transformer } \\
\text { protection } \\
\text { operates or not }\end{array}$ & $\begin{array}{c}\text { Bus protection } \\
\text { operates or not }\end{array}$ & $\begin{array}{c}\text { Breakers break } \\
\text { position }\end{array}$ \\
\hline$F_{l}$ & No & No & Yes & Yes & No & $k, j, l$ \\
$F_{I}$ & Yes & No & No & No & No & No \\
$F_{l}$ & No & Yes & No & No & Yes & $n, l, a, c$, \\
$F_{2}$ & No & No & Yes & No & No \\
$F_{3}$ & No & No & No & No & No \\
\hline
\end{tabular}

\section{Conclusion}

Aiming at a series of problems, such as protection misoperation and refusing to operate, which may lead to the decline of power system stability due to the lack of measurement information of smart substation, a station protection method which can effectively eliminate the influence of transformer inrush current and bus CT saturation is proposed when the measurement information of Substation and bus connecting line is missing.

In addition, the great harm that the lack of measurement information in substation may bring is expounded, the urgency and necessity of dealing with the lack of measurement information is illustrated, the characteristics and limitations of research at home and abroad is summarized, and theoretical analysis on the current synthesis method of the missing point of measurement information based on Kirchhoff current law is made, which proves that this method can not effectively eliminate the adverse effects of transformer inrush current and bus CT saturation on the protection. The specific countermeasures and tripping strategies of the station protection is given in this paper. The method proposed in this paper is simple, easy to implement, and can be applied to various topologies of power grid.

The substation model is built on PSCAD / EMTDC simulation platform, and the proposed protection method is verified. The results show that the proposed substation protection method can not only accurately eliminate the interference of transformer inrush current and bus CT saturation, but also ensure the correct operation of differential protection for different types of faults and improve the stability of substation when the measurement information is missing.

\section{Acknowledgement}

Fund project: Science and technology project funding of China Southern Power Grid Company (036000KK52200011)

\section{References}

1. Huang Tianyi. Research on fault characteristic and protection scheme of distribution network with distributed generation. [D]. Beijing: North China Electric Power University, 2016.

2. Ma Jing, Shi Yuxin, Ma Wei, et al. Substation backup protection based on current fusion reliable factor. [J]. Power System Protection and Control, 2015, 43(4): 19.

3. Sun Weinan. Research on substation protection scheme based on bay level protective relays. [D]. Beijing: North China Electric Power University, 2016.

4. Yan Yang. Study of centralized protection based on digital substation. [D]. Wuhan : Huazhong Universtiy of Science and Technology, 2011.

5. Li Zhenxing, Yin Xianggen, Zhang Zhe, et al. Wide area protection algorithm based on multi-information fusion. [J]. Automation of Electric Power Systems, 2011, 35(9) : 14-18.

6. You Hao, Shi Hengchu, Yang Yuanhang, et al. Intelligent fusion diagnosis method for multi-source information of power grid fault based on improved D$\mathrm{S}$ evidence theory. [J]. Guangdong Electric Power, 2020, 33(11) : 16-25.

7. Jiang Rongzhou. Research on wide area backup protection based on centralized decision-making. [D]. Wuhan: Huazhong Universtiy of Science and Technology, 2011.

8. Wei Jidong, Wang Runzhi, Jin Yuwei, et al. An integrated protection algorithm of intelligent substation. [J]. Journal of Changchun University of Science and Technology( Natural Science Edition), 2017, 40(3) : 139-142.

9. Jiang Yiwen, Peng Mingyang, Ma Kai, et al, Evaluation method for power transformer conditions based on multi-source heterogeneous data fusion. [J]. Guangdong Electric Power, 2019, 32(9) : 137-145.

10. Xia Wenlong. Study on adaptive protection considering wide-area information. [D]. Wuhan: Huazhong Universtiy of Science and Technology, 2012. 